\title{
Políticas de comunicación y medios en entornos de convergencia en América Latina: una aproximación a los casos de Uruguay y Colombia ${ }^{1}$
}

\author{
Recibido: 27 de agosto de 2018 \\ Aceptado: 9 de octubre de 2018 \\ Publicado: 28 de noviembre de 2018
}

\author{
Federico Beltramelli \\ federico.beltramelli@fic.edu.uy \\ Universidad de la República (Uruguay) \\ Débora Pérez Serna \\ deboraperez2002@yahoo.com.mx \\ Investigadora independiente (Colombia) \\ Diana Lombana Herrera \\ lombana.dianapatricia@gmail.com \\ Investigadora independiente (Colombia)
}

Resumen: Este artículo aporta reflexiones sobre la agenda de investigación situada en la intersección de cambios normativos procesados y el avance de nuevas tecnologías convergentes para el sector de los servicios de comunicación audiovisual (SCA). Se parte de una sistematización de dos casos (Colombia y Uruguay) en los cuales se identifican tendencias de políticas de comunicación y se evidencian similitudes y diferencias de cada país, así como en las arquitecturas institucionales y los cambios regulatorios recientes, con la finalidad de revisar los desafíos y perspectivas para formular políticas de comunicación y medios en el marco de las transformaciones tecnológicas.

Palabras clave: Convergencia, arquitecturas institucionales, marcos regulatorios.

Abstract: This paper contributes for the research agenda located at the intersection of processed normative changes and the advancement of new convergent technologies for

1 Algunos pasajes de este artículo fueron presentados y discutidos en el marco del GT 10 de ALAIC 2018, organizado en San José de Costa Rica. 
the Audiovisual Communication Services (ACS) sector. It is based on a systematization of two cases (Colombia and Uruguay) which trends of communication policies are identified, evidencing similarities and differences of each country, institutional architectures and recent regulatory changes in order to review the challenges and perspectives for formulate communication and media policies within the framework of technological transformations.

Key words: Convergence, Institutional Architectures, Regulatory Frameworks.

\section{Introducción}

Los procesos de cambios tecnológicos en el sector de los SCA (tanto como de las telecomunicaciones, en los primeros quince años del siglo XXI) conforman una arena permanente en las investigaciones en el campo de la comunicación. La afectación que estos cambios económicos y culturales provocan en países emergentes (con una historia socioeconómica pautada por procesos de bienestar limitados, y con prácticas neoliberales ${ }^{2}$ de desmontaje estatal) mantienen un alto impacto en las estructuras económicas y culturales asociadas a los SCA, además de configurar un espacio demandante para los entornos regulatorios de los Estados nacionales.

Las experiencias no resultan unánimes, debiéndose abordar los estudios caso a caso. Cada país tributa a su particular historia y trayectorias en relación con la evolución de estos fenómenos. Lo que sí resulta un común denominador (tanto para países emergentes como centrales) es la complejidad que agrega a un panorama de medios signado por procesos de alta concentración, regulación acotada y Estados con capacidades limitadas para garantizar un conjunto de derechos ciudadanos básicos.

De este conjunto dinámico de fenómenos, algunas investigaciones vienen dando cuenta (tanto en el entorno latinoamericano como en el global) de lo que implica considerar un campo activo en la intersección de los estudios sobre las tecnologías aplicadas al campo de la comunicación y como estos cambios vienen asociados a respuestas regulatorias mediante una matriz de políticas públicas sectoriales (Castells, 2009; Becerra, 2000, 2015; Bustamante, 2003; Monje, 2013, Bizberge, 2012).

Sobre esta tensión, no está demás incluir una consideración sobre los procesos de integración económico-financieros que terminan por configurar un mapa complejo que excede claramente los límites del Estado-nación y que han sido extensamente estudiados por algunos autores (Becerra, 2014; Castells, 2009; Mattelart, 2006; García Canclini,

2 Las discusiones críticas, evaluativas y, sobre todo, políticas (en relación con el Estado de bienestar y a su implementación desde la etapa posbélica) ambientan algunas dimensiones analíticas sobre el Estado como figura central o marginal que trascienden la discusión concreta sobre el caso y que delinean las distintas propuestas en torno al par de bloques que conforman el Estado y la sociedad, y terminan por implicar (de alguna manera u otra) a una multiplicidad de actores y arenas que hacen de este duopolio una cuestión compleja y omnipresente (Álvarez-Miranda, 1996). 
2001; Wolton, 2003; Mastrini, De Charras \& Fariña, 2013), extremos que cobran mayor consideración si miramos el panorama convergente actual.

Asumimos que los regímenes de competencia en el sector de los servicios de SCA y telecomunicaciones han cambiado por la vía de integraciones comerciales y productivas y esto ha determinado un alto grado de hibridez con relación a los rubros que involucran. El nuevo paisaje que se abre con los procesos de convergencia tecnológica en la industria infocomunicacional no solo desafía a los Estados, sino también a configuraciones comerciales locales que, por la vía de comportamientos asociados al statu quo reinante, ven cuestionados sus modelos productivos y de negocios (Wohlers, 2008; Castells, 2009; Mattelart \& Mattelart, 1997; Mosco, 1996, 2011; McChesney, 2004). De algún modo podríamos pensar en un formato de doble convergencia, que combina los procesos tecnológicos descritos y la integración de capitales globales, de forma vertical y transversal, en el mercado de la industria (Becerra y Mastrini, 2007, 2009; Apreza, 2002).

\section{Arenas y movimientos}

Los ecosistemas de medios son afectados de forma combinada tanto por las regulaciones como por cambios en los procesos tecnológicos. En la esfera de SCA, el fenómeno que ha cobrado mayor interés académico es el de la convergencia de medios, servicios y acceso. Asimismo, la transnacionalización de la industria de la información y de contenidos (sumada al crecimiento del consumo de bienes culturales y de sus canales de circulación) son dimensiones de análisis inminentes en la realidad actual (Albornoz, 2011; Bustamante, 2003; Becerra, 2003; Sierra, 2011). La fecundidad o inocuidad de los marcos regulatorios (aplicados a servicios tradicionales para la regulación de estrategias de servicios y consumos) son cada vez más ubicuos y más difíciles de abordar desde una estrategia de investigación que no parta de la consideración de una estrategia comparada e interdisciplinaria.

Como premisa, reconocemos que toda incorporación tecnológica cuenta con un paradigma político y social de base, el cual ejerce su acción de forma mayormente explícita o implícita. Podemos asumir la existencia de lógicas subyacentes en la aplicación de soluciones tecnológicas y la aplicación de marcos regulatorios, conformando un blending complejo entre políticas públicas, paradigmas de funcionamiento, actores comerciales privados y públicos intervinientes, donde todos empujan los límites y las definiciones de alcance (Freedman, 2008; Garnham, 2000).

Con referencia a la convergencia tecnológica y la agenda regulatoria de las telecomunicaciones, Wohlers (2008) planteaba que la convergencia tecnológica, en la práctica, implica una segunda transformación del modelo regulatorio tradicional de las telecomunicaciones. Luego de la primera transformación (procesada en la década de los años ochenta y noventa, con la ruptura de los monopolios públicos de las telecomunicaciones), Wohlers propone una conceptualización en términos de segunda fase de reformas. Este autor entiende que las posibilidades tecnológicas asociadas al protocolo IP (tanto como la posibilidad técnica de embeber contenidos a partir de los procesos de digitalización y como la ampliación de redes 
y dispositivos de acceso) han generado un segundo gran cambio estructural, que supera al movimiento privatizador ya procesado en el sector, que en su momento

constituyó un movimiento de naturaleza estructural, de gran impacto político y económico, con inclusión de instituciones nuevas para el sector, como la OMC. Pero, no alteró los fundamentos técnicos de las clasificaciones de servicios y la emisión de licencias, manteniendo además la separación institucional y legal entre los diferentes sectores TIC, particularmente entre los de telecomunicaciones y de radiodifusión. De cierta forma, la convergencia tecnológica plantea una nueva (segunda) transformación, inclusive de naturaleza más conceptual, en los fundamentos técnicos y económicos, no solo de los servicios exactamente, sino también en la propia normativa de clasificación de servicios que sustenta el modelo regulatorio convencional (Wohlers, 2008, p. 6).

Para el mencionado autor, las tecnologías convergentes complejizan algunos ejes de desarrollo y consumo de contenidos, debido a que espacios controlados por el emisor dejan lugar al contexto de recepción, ya sea por las posibilidades atribuidas a la interactividad o a la personalización del consumo; sea por la posibilidad tecnológica de la ubicuidad y el manejo multiplataforma en el acceso de contenidos. Otra de las bifurcaciones que señala como marca de identidad de la convergencia es la agregación de un conjunto de externalidades a las redes de SCA y telecomunicaciones, hasta este momento inexistentes. Asimismo, advierte sobre la necesidad de considerar el cambio procesado en la relación de costos en la trasmisión y conmutación. Los costos de conmutación y trasmisión, así como la inversión en infraestructura, funcionaron muchas veces como la base argumental para el establecimiento de monopolios naturales (muchos de ellos en manos de los Estados nacionales) en el terreno de las telecomunicaciones.

En la actualidad (debido a la exponencial solicitud de servicios, así como por la disminución de costos), la base argumental de costos enterrados ha dejado lugar a otras discusiones mucho más refinadas sobre la estructura de mercado que, por cierto, configuran un espacio muy dinámico y con barreras que intentan romperse en la aspiración de cuotas de mercados, tanto por actores nacionales como por mercados globales en esquemas de convergencia en la distribución de contenidos (Wohlers, 2008, p. 17).

La reformulación de estas reglas de juego puede traer asociado el establecimiento de barreras de acceso, como un reflejo automático de preservación de mercados. Los actores en los entornos nacionales buscan un "retorno" del Estado, como forma de preservación ante una oleada global de mercados convergentes. La implicancia de estos movimientos en la formulación de políticas públicas puede darse por la imposición de barreras discriminatorias en el tratamiento sectorial o por la modificación de la aplicación reglamentaria en el uso y alcance de las licencias radioeléctricas.

Los modos de preservación de mercado en el entorno latinoamericano no pasaron de ser intentos nacionales. Sin perjuicio de mantener algunos acuerdos estratégicos regionales, cada país viene aplicando fórmulas particulares. Esto último agrega mayor tensión en los diseños normativos en el eje regulación por tecnología o servicio, ya que tratamientos 
regulatorios dispares y por país no aseguran una comparecencia ordenada en los debates internacionales, mucho menos un marco regional de protección estratégica.

Monje (2013) plantea escasos avances en el entorno mercosuriano en materia de políticas audiovisuales, por citar un ejemplo. El cuidado de algunas demarcaciones tradicionales en los ecosistemas de medios se ve atacado por regulaciones por momentos inocuas y por procesos de convergencia que combinan cambios tecnológicos a partir de "la consolidación de nuevas redes digitales que conmocionan, ellas también, las certezas de la ciudadela mediática tradicional" (Becerra, 2015: 158). De este modo, los arreglos de entrecasa ceden ante un aluvión digital de contenidos. El esfuerzo de los sectores tradicionales por mantener el statu quo (fuertemente atacado por las regulaciones de la década progresista latinoamericana) ahora vuelve a ser desafiado y "afronta un torbellino de transformaciones para cuyos desafíos no halla, por el momento, respuestas novedosas” (Becerra, 2014).

A partir de estos antecedentes, presentamos una descripción y análisis de las políticas y marco regulatorio aplicados para los casos de Uruguay y Colombia a los efectos de tomarlos como antecedentes e hitos constatables. La indagación gira en torno a cuatro ejes identificados: a) aspectos coyunturales de cada país, b) observación de cambios regulatorios recientes, $c$ ) comprensión de la arquitectura institucional y e) identificación de retos y desafíos.

\section{Caso uruguayo}

En el marco de la irrupción de tecnologías en el campo de los servicios de comunicación, las políticas públicas implementadas en Uruguay entre los años 2005 y 2014, orientadas al sector de los SCA, carecieron de un tratamiento convergente. Entre los años 2005 y 2014 gobernó el Frente Amplio, una fuerza política que se ubica en el arco de los gobiernos progresistas latinoamericanos de inicios del siglo XXI (Saintout \& Varela, 2013; Badillo, Mastrini \& Marenghi, 2015; Gómez, 2011). De forma concurrente con otros gobiernos progresistas de Latinoamérica ${ }^{3}$ (Argentina, Bolivia, Ecuador, Uruguay, Venezuela) en

3 Siguiendo a Santander (2014), podemos identificar en los países de frente progresista o populares latinoamericanos procesos de revisionismo legislativo y regulatorio en materia de SCA. En Argentina, en el año 2009, se votó la ley de SCA; en Uruguay, en 2008, con la ley de Radiodifusión Comunitaria. Como en 2014, con la aprobación de la ley de SCA, se produjeron cambios históricos en la regulación de SCA. En Venezuela, los cambios aparecieron más temprano, con la aprobación en el año 2000 de la ley Orgánica de Telecomunicación, el reglamento de Radiodifusión Sonora y TV Abierta Comunitaria en el año 2002 y, en el año 2005, se aprobaba la ley de Responsabilidad Social en Radio y Televisión. En Bolivia, en el año 2011, se promulgó la ley General de Telecomunicaciones, Tecnologías de Información y Comunicación y, en Ecuador, en el año 2014, se efectivizó la ley Orgánica de Comunicación. En Brasil, se llevó adelante en 2009 la I Conferencia Nacional de Comunicación (Confecom) y, en 2014, se aprobó el "Marco civil de internet". De esta forma, el ciclo de reformas que se abría con Venezuela se cerraba con Uruguay con la aprobación de la ley sobre SCA. Algunos autores señalan que estas reformas conformaron un cuerpo de soluciones simultáneas donde, en algunos casos, se evidencian grandes coincidencias programáticas (Becerra, 2015; Santander, 2014). 
este período se aplicaron reformas inéditas en materia de servicios de comunicación (radiodifusión), aunque se mantuvo incambiada la regulación para el sector de las telecomunicaciones.

Históricamente, en Uruguay se evidenció una división tácita entre los SCA(mayoritariamente operados por privados, asociado a un régimen altamente concentrado) y el de las telecomunicaciones, operadas bajo un régimen cuasi monopólico por la empresa estatal ANTEL $^{4}$, hasta la irrupción de las tecnologías de conectividad móvil (telefonía celular o móvil). Esta situación se mantuvo en el período reciente 2005-2014, aunque fue desafiada por algunas modificaciones regulatorias, como la Ley de Radiodifusión Comunitaria (LRC) $\mathrm{N}^{\circ} 18.232$, del año 2008, el otorgamiento de licencias de TV digital (2012-2013) y la ley $\mathrm{N}^{\circ}$ 19.307 de Servicios de Comunicación Audiovisual (LSCA) del año 2014.

Ninguno de estos cambios regulatorios y legales mantuvo un tratamiento convergente. En Uruguay, con anterioridad a los períodos de gobierno del Frente Amplio (2005-2014), se observaban procesos de concentración y oligopolio privado en los servicios de comunicación audiovisual; una producción audiovisual incipiente y sin pantalla; radios comunitarias ilegales; medios públicos e institucionalidad regulatoria débiles y dependientes del gobierno; una regulación parcial y desagregada en un cuerpo legal y regulatorio disperso, así como una división tácita entre el sector de los SCA y las telecomunicaciones a nivel de servicios.

A partir del año 2005 se evidencian cambios regulatorios a partir de la adopción de la norma de TV digital: primero la DVB-T (2007) y luego la ISDB-T (2010) en consonancia con la región. Se aprueba la LRC (2008) y se establece el decreto regulatorio sector comercial de radiodifusión (2008). Además, se aprueban los decretos para el proceso de adjudicación TV digital (2012-13) y se vota la LSCA (2014).

Como resultado de este cuerpo inédito de reformas se observaron resultados parciales, como el promovido por la LRC, que generó la legalización de 140 radios comunitarias. Asimismo, se destaca la promoción de prácticas de adjudicación transparentes y con participación social para el sector comercial de radiodifusión. En relación con el proceso de adjudicación de TV Digital, se reconocen tres sectores: $a$ ) el comunitario, $b$ ) el comercial y c) el estatal-público, aunque en diciembre de 2017 existía un $80 \%$ de lo adjudicado sin uso real. En relación con la ley "madre" de los SCA, se constata una tardía y casi nula aplicación. Recién a finales del año 2017 comienza a discutirse su reglamentación y se instituye parcialmente la nueva institucionalidad prevista a los efectos de la regulación de radiodifusión.

4 La Administración Nacional de Telecomunicaciones (ANTEL), es la empresa de telecomunicaciones del Estado uruguayo. Fue por momentos regulador parcial de las telecomunicaciones en el país y actualmente es la empresa de mayor facturación en Uruguay en su rubro y una de las mayores, tomando en consideración a otros rubros comerciales. 
No existe en toda la normativa reseñada una vocación de tratamiento convergente. Deliberadamente se excluye de la ley que regula a los SCA (Ley $\mathrm{N}^{\circ}$ 19307), cualquier posibilidad en ese sentido.

No son objeto de regulación en la presente ley: los servicios de comunicación que utilicen como plataforma la red de protocolo Internet. Las redes y servicios de telecomunicaciones que transporten difundan o den acceso a un servicio de comunicación audiovisual, así como los recursos asociados a esos servicios y los equipos técnicos necesarios para la recepción de estos, que estarán sujetos a lo dispuesto en la normativa sobre telecomunicaciones. Los servicios de telecomunicaciones y de comercio electrónico a los que se accedan a través de un servicio de comunicación audiovisual (Art. 1 de la Ley No 19307 literales a, b y c).

De este modo se mantiene una doble escena en Uruguay (Beltramelli \& Kaplún, 2017), donde las telecomunicaciones y los SCA funcionan en su desempeño comercial y regulatorio como compartimentos estancos. Se evidencian cambios inéditos en los SCA, donde existieron cambios regulatorios y políticas explícitas de transformación, las que por otra parte han tenido productividad media (LRC) o baja (TDT-LSCA). Las Telecomunicaciones, por su parte, se mantienen sin cambios regulatorios y con poca explicitación de políticas, con productividad media y alta. El mercado de televisión comercial se mantiene de forma similar (televisión abierta) al periodo que antecede a las reformas regulatorias.

\section{Caso colombiano}

En Colombia, la Constitución Política de 1991 enmarca una serie de principios de comunicación y cultura entre los que se encuentran la promoción de la diversidad étnica y cultural ${ }^{5}$ que fueron determinantes en la formulación de políticas y regulaciones sobre los medios de comunicación comunitaria y que ayudaron a formalizar tendencias informales y marginales de uso de la radio y televisión en ámbitos locales y comunitarios que la utilizaban como medio de expresión y reivindicación popular ayudados por parlantes, perifoneo y estaciones ilegales de radio.

El primer gran hito de radiodifusión sonora comunitaria en Colombia se presentaría con el extinto decreto 1695 del 3 de agosto de 1994, así como los decretos 1445, 1446 y 1447 de 1995. Estos, en gran medida, surgieron como amnistías y necesidad de legalización de operadores informales. En 1997 se adjudican alrededor de 500 licencias para el servicio de radio comunitaria y Colombia se vuelve un referente de políticas de comunicación

5 La promoción y el fomento del acceso a la cultura de todos los colombianos (art. 70); la libertad de expresión e información y de fundar medios de comunicación (art. 20); la intervención estatal en el espectro electromagnético con el fin de evitar prácticas monopolísticas en el uso del espectro radioeléctrico y garantizar el pluralismo informativo y la libre y leal competencia (art. 75). Principios determinantes en la formulación de políticas y regulaciones sobre los medios de comunicación comunitaria que se empiezan a desencadenar en los noventas. 
comunitaria en América Latina. El Ministerio de Comunicaciones se vuelve el ente regulador de los servicios de telecomunicaciones en Colombia.

En 1997 se crea el Ministerio de Cultura gracias al espacio que abre la Constitución de 1991, aspecto que genera gran dinamismo y articulación con otros ministerios como el de Comunicaciones y Educación, en función del desarrollo de programas de fortalecimiento de la comunicación. Aparecen además nociones, tales como la comunicación pública, los servicios de interés público y se da énfasis a la promoción de la educación y la cultura que se reflejan en el establecimiento de franjas para niños y jóvenes entre otras políticas de contenidos audiovisuales.

Años después de la adjudicación de licencias de radio comunitaria se realizan varias mesas de trabajo encabezadas por los ministerios de Comunicaciones y de Cultura que contribuyen a la creación del decreto 1981 de 2003 que reglamenta el Servicio Comunitario de Radiodifusión Sonora, adicionando nuevos lineamientos para el fortalecimiento de las radios como las Juntas de Programación, la implementación de esquemas de rendición de cuentas periódica y la creación de instrumentos de planificación como los "manuales de estilo". En paralelo a este proceso se crea la Comisión Nacional de Televisión (CNTV), ente rector del diseño y ejecución de las políticas de televisión mediante la Ley 182 de 1995. Dicho ente expide el Acuerdo 006 de 1996 y el del 6 del 1999, en los que se establecen condiciones de operación de la televisión comunitaria.

Diez años después, en 2009, se crea la Ley 1341 (Ley TIC) que transforma el Ministerio de Comunicaciones (establecido desde 1953) en el Ministerio de las Tecnologías de la Comunicación y la Información (MinTIC), buscando una reorganización institucional que responda a las transformaciones de las tecnologías de la información en el mundo y a los fenómenos de convergencia de los servicios de telecomunicaciones. El artículo primero de esta ley define el marco general de las tecnologías de la información y la comunicación, en materia de cobertura, calidad, promoción de la inversión y desarrollo de las tecnologías; el uso eficiente de redes y del espectro radioeléctrico y determina las potestades del Estado en relación con la planeación, la gestión, la administración (adecuada y eficiente) de los recursos, regulación, control y vigilancia, facilitando el libre acceso a la sociedad de la información. El parágrafo de esta norma exceptúa el servicio de televisión y el servicio postal, los cuales continúan rigiéndose por las normas especiales pertinentes, con las excepciones específicas previstas en la 1341 (art. 72).

Resultado de lo anterior se crean como unidades especiales del sector la Comisión de Regulación de las Comunicaciones (CRC) como órgano encargado de promover la competencia y regular los mercados de las redes y los servicios de comunicaciones, con el fin de velar por la prestación eficiente de los servicios frente a sus usuarios; y la Agencia Nacional del espectro (ANE) como responsable de realizar la planeación, vigilancia y control del espectro radioeléctrico en Colombia, así como brindar la asesoría técnica para su gestión. Igualmente, como entidades vinculadas se encuentran la sociedad de Radio y Televisión de Colombia (RTVC), las empresas de servicios públicos y servicios postales y como entidades adscritas encontramos el Fondo de Tecnología de la Información y las Telecomunicaciones (FONTIC). 
En cuanto a la nueva arquitectura institucional, se determinan una serie de responsabilidades que se observan en Colombia de la siguiente manera:

- A través de la Ley 182 de 1995, se mantiene vigente el régimen de concesión de los servicios de televisión y radiodifusión sonora por parte del Estado, que tiene la reserva de la prestación de los servicios y autoriza a particulares a través de contratos de concesión o licencias.

- El artículo 12 de la Ley 1507 de 2012 dio vida a la Autoridad Nacional de Televisión $\left(\mathrm{ANTV}^{6}\right)$ una entidad de carácter técnico responsable de la expedición de la regulación que determina las condiciones de explotación del servicio de televisión, responsable de la contratación y adjudicación de las concesiones y licencias y espacios de televisión.

- El Ministerio de Tecnologías de Información y Comunicaciones mantiene (a través de la Ley 1341 de 2009) las funciones de regulación, vigilancia y control de la radiodifusión sonora.

- La Agencia Nacional del Espectro, a partir de la ley 1341, tiene funciones de planeación, vigilancia y control del espectro radioeléctrico en Colombia, así como brindar la asesoría técnica para su gestión, de administración y gestión del espectro de las Tecnologías de Información y Comunicación y del Servicio de Radiodifusión sonora (art. 26).

- La Comisión Nacional de Comunicaciones tiene funciones de regulación de los servicios de redes y telecomunicaciones.

- $\quad$ El Decreto 993 de 2012, a través del cual se establece el Tratado de Libre Comercio con (TLC) suscrito entre Colombia y los Estados Unidos de América, abre la posibilidad de acceso total a los servicios de telecomunicación cualquiera sea su naturaleza. Existe la obligación de permitir el acceso total a los servicios de telecomunicaciones bien sea públicas o privadas, consecuencia de esto es que las programadoras no nacionales lograrán prestar sus servicios en el territorio nacional sin mayor restricción.

\section{Desafíos actuales para Colombia}

Podemos observar que la Ley 1341 de 2009 que crea el MinTIC prevé un contexto de convergencia y articula sectorialmente a varias entidades encargadas de regular el sector de las telecomunicaciones en Colombia. Asimismo, esta ley responde a necesidades establecidas en el TLC con Estados Unidos e incluso genera condiciones para darle viabilidad al mismo. Sin embargo, dicha ley y sus consecuentes actualizaciones facilitan prioritariamente la inversión extranjera, así como la prestación de servicios integrados por parte de multinacionales o grandes inversionistas.

6 La creación de la ANTV aparece como la figura que reemplazaría a la Comisión Nacional de Televisión (CNTV) que fue creada mediante la Ley 182 de 1995. 
La actual arquitectura evidencia la duplicidad de funciones regulatorias entre MinTic, la ANTV y la CRC que entran en contradicción con la dinámica de las nuevas tecnologías de información y comunicación que integran para los ciudadanos y consumidores servicios de telecomunicaciones y contenidos. Por su parte, en el sistema de concesiones, la habilitación y facilidades en la prestación de servicios (para nuevos proveedores de redes) ponen en desventaja y limitan las posibilidades de acceso a proveedores de servicios de telecomunicaciones tradicionales como la radio y la televisión. Aspecto que también deja de lado a las mini pymes, a productores independientes y a los medios de comunicación locales y comunitarios.

A pesar de estas facilidades para los prestadores de servicios de redes, se mantienen legislaciones como el Decreto 1161 de 2010, Documento CONPES 3506 de 2008, Sentencia de la Corte Constitucional 460 de 2006, incluso en regulaciones anteriores del Ministerio de Cultura, a través de la Dirección de Comunicaciones ${ }^{7}$, que están desarticuladas e incluso son contradictorias con la nueva regulación TIC.

Si bien es cierto que las trasformaciones tecnológicas implican la aparición de nuevos actores en el escenario de servicios de telecomunicaciones (para múltiples pantallas) se corre el riesgo de privilegiar un enfoque regulatorio hacia la infraestructura con una mirada netamente económica y de desregulación, que deja de lado políticas y programas para la promoción de la diversidad que estimulen el desarrollo social del país, manteniendo el espíritu de la Constitución de 1991.

La Ley TIC 1341 ha evidenciado la prioridad por garantizar cobertura de servicios universales de telecomunicaciones en el territorio colombiano y olvida políticas de contenidos audiovisuales carácter comunitario, universitario y público. De acuerdo con lo anterior, es fundamental priorizar la libertad de expresión y el pluralismo en las dinámicas regulatorias de tal manera que garanticen el acceso equitativo a los servicios de telecomunicaciones, prevengan el establecimiento de monopolios y velen por que efectivamente respondan a su finalidad como espacios de participación y expresión de la diversidad.

El análisis publicado por Mastrini \& Becerra (2016) evidencia como los fenómenos de convergencia tecnológica han agudizado los índices de concentración a la propiedad de los servicios de telecomunicación. De ahí que cuatro empresas dominen el 95\% del mercado en la región. Asimismo, en los últimos años, para el caso de Colombia, se ha incrementado la suscripción y acceso a televisión paga, la cual, en su mayoría, está en manos de cuatro empresas que tienen el $92 \%$ del mercado.

7 Decreto 1746 de 2003 que actualiza las funciones de la Dirección de Comunicaciones del Ministerio de Cultura de Colombia y le atribuye la formulación de "políticas que contribuyan al ejercicio cotidiano de una cultura democrática y al reconocimiento de la multiculturalidad del país y del mundo a través de los medios" y otras como el Plan Nacional de Cultura 2001-2010, el Compendio de Políticas Culturales de 2010, la actualización de los lineamientos de políticas de comunicación y cultura de 2014 de la Dirección de Comunicaciones del Ministerio. 
Para la UNESCO (2008), la concentración influye en las dinámicas culturales y sociales de los países, afecta el desarrollo de los medios y la democracia; así lo plantea en el documento de indicadores de desarrollo mediático, pues podría limitar el acceso a la información, a la libertad de expresión, a la diversidad de actores que participan en la producción de contenidos mediáticos, entre otros aspectos. La UNESCO expresa la importancia del acceso a los medios como un aspecto fundamental para la libertad de expresión pues constituyen una "plataforma" para ejercer este derecho.

\section{Hacia una nueva agenda}

En Latinoamérica, como lo han indicado algunos investigadores, el camino estuvo a contramano de lo definido en los países "centrales". Mientras en los grandes centros se optaba por una distensión de los marcos regulatorios de cara a la convergencia, en América Latina se emprendían regulaciones que atendían a lo sectorial de los SCA. Esto último se cumplió en la primera quincena del siglo XXI en varios países de la región: Argentina, Brasil, Ecuador, Uruguay y Venezuela.

El objeto de estas modificaciones normativas pocas veces intervino en el nuevo campo que se abría a partir de los procesos de convergencia. Esto se explicaba, en parte, por la comparecencia que evidenciaba el sector de las telecomunicaciones en el marco de OMC a diferencia del sector de los SCA y la cláusula cultural, así como también por distintas realidades locales y las estrategias de implementación definidas en los distintos países donde, en muchos casos, parecía razonable ir de la parte al todo.

Al esfuerzo acumulado en los nuevos marcos regulatorios (que mantenían como objetivos el asegurar un nuevo marco de derechos ciudadanos) se le anteponían cambios en los modelos de negocio, desintermediación entre la estructura mediática tradicional y las formas de consumo, lo que para algunos trajo aparejada una crisis de objeto en las regulaciones.

La política de medios, que se ocupó de la regulación de un sector cardinal en la configuración de las sociedades en el siglo XX, y en particular de la industrialización, masificación y mercantilización de los medios de comunicación y de las industrias culturales, está siendo desafiada por la crisis del objeto mismo al que está consagrada. En la actualidad, el sector de los medios protagoniza un proceso inédito de convergencia digital con las telecomunicaciones y las redes informáticas. El resultado final de ese proceso es aún incierto, ya que se halla en pleno desarrollo (Becerra, 2014, pp. 73-74).

Las nuevas articulaciones desarrolladas por los procesos de convergencia también admiten arenas de lucha entre posicionamientos que observan los procesos de asignación y reserva de espectro que algunos marcos normativos han asegurado. De modo que las disputas las debemos alojar entre políticas nacionales de comunicación, gobernanza internacional e instituciones internacionales. Califano \& Baladrón (2013) refieren a la gobernanza de Internet como un encuadre complejo y con dependencia al enfoque, ya que (desde la 
óptica de las telecomunicaciones) la informática o los derechos humanos, los resultados pueden ser distintos. De esta definición se desprende un encuadre de análisis y discusión no nacional y no jerarquizada, sino más bien una estructura horizontal de "interacción entre las instituciones políticas, sociales y privadas, no solo del ámbito nacional, sino también supranacional" (Califano \& Baladrón, 2013, p. 7).

El eje de discusión importante para los países implica que converjan contenidos y tecnología. Esto no significa que sea menos importante diferenciar y hacer políticas de contenidos que interpele a las audiencias no sólo como consumidores de servicios, sino como ciudadanos usuarios de servicios públicos. Es evidente el tratamiento que este tema implica para la economía de medios: la convergencia es una necesidad de desarrollo tecnológico y de economía digital, pero en ese contexto se debe garantizar el acceso equitativo, la propiedad plural y la diversidad.

En el caso colombiano, se percibe una tendencia a priorizar la liberalización de mercados, la apertura a la economía de lo digital y, por tanto, la definición de una política convergente mantiene el riesgo de procesos de concentración y de falta de pluralidad en el ecosistema de medios. Para el caso uruguayo, observamos cambios inéditos con poca aplicación en las regulaciones sobre SCA y escasa o nula vocación convergente en el tratamiento regulatorio y la institucionalidad a cargo de la gestión y control.

En los dos casos analizados se evidencia una desconexión entre las instituciones, las arquitecturas institucionales propuestas son estructuras rígidas o repetidas, que de alguna manera evidencian la dificultad para pensar regulaciones convergentes sin irse o a un extremo a otro.

\section{Referencias}

Albornoz, L. (Comp.) (2011). Poder, medios y cultura. Una mirada crítica desde la economía política de la comunicación. Buenos Aires: Paidós.

Alvarez-Miranda, B. (1996). Dilemas del Estado de bienestar. Madrid: Visor.

Apreza, S. (2002). Concentración de medios de comunicación versus pluralismo informativo externo. Biblioteca Jurídica Virtual, 63-85. Recuperado de https:// archivos.juridicas.unam.mx/www/bjv/libros/5/2444/7.pdf

Astrini, G., De Charras, D. \& Fariña, C. (2013). Nuevas formas de regulación internacional y su impacto en el ámbito latinoamericano. En G. Mastrini, A. Bizberge y D. de Charras (Eds.), Las políticas de comunicación en el siglo XXI. Nuevos y viejos desafios. Buenos Aires: La Crujía

Badillo, A., Mastrini, G. \& Marenghi, P. (2015). Teoría crítica, izquierda y políticas públicas de comunicación: el caso de América Latina y los gobiernos progresistas. Comunicación y Sociedad, 24, 95-126. Recuperado de http://www.scielo.org.mx/ scielo.php?script=sci_arttext\&pid=S0188-252X2015000200005 
Becerra, M. (2000). De la divergencia a la convergencia en la sociedad informacional: fortalezas y debilidades de un proceso inconcluso. ZER. Revista de Estudios de Comunicación, 5(8). Recuperado de http://www.ehu.eus/ojs/index.php/Zer/ article/view/17418/15193

Becerra, M. (2003). Sociedad de la información: proyecto, convergencia, divergencia. Bogotá: Norma.

Becerra, M. (2014). Medios de comunicación: América Latina a contramano. Recuperado de http://nuso.org/articulo/medios-de-comunicacion-america-latina-a-contramano/

Becerra, M. (2015). De la concentración a la convergencia. Políticas de medios en Argentina y América Latina. Buenos Aires: Paidós.

Becerra, M. \& Mastrini, G. (2007). Presente y tendencias de la concentración de medios en América Latina. Zer, 22, 15-40. Recuperado de http://www.ehu.eus/ojs/index. php/Zer/article/download/3666/3298

Becerra, M. \& Mastrini, G. (2009). Los monopolios de la verdad. Buenos Aires: Prometeo.

Becerra, M. \& Mastrini, G. (2010). Concentración de los medios en América Latina: Tendencias de un nuevo siglo. Contratexto, 18, 41-64. Recuperado de http:// fresno.ulima.edu.pe/sf/sf_bdfde.nsf/OtrosWeb/CONT18BECERRA/\$file/03contratexto $18 \% 20$ BECERRA.pdf

Beltramelli, F. \& Kaplún, G. (2017). Políticas de comunicación en Uruguay: el país de la doble escena. Cartagena: IAMCR.

Bizberge, A. (2012). El impacto de Internet en los modelos de negocio de la industria televisiva en América Latina. Un estudio de la convergencia tecnológica en cinco grupos multimedia. Buenos Aires: UNQ.

Bustamante, E. (2003). Hacia un nuevo sistema mundial de comunicación. Barcelona: Gedisa.

Califano, B. \& Balandrón, M. (2013). La regulación de Internet: formas emergentes de gobierno privado. En G. Mastrini, A. Bizberge y D. de Charras (Eds.), Las políticas de comunicación en el siglo XXI. Nuevos y viejos desafios. Buenos Aires: La Crujía.

Castells, M. (2009). Comunicación y poder. Madrid: Alianza.

Freedman, D. (2008). The Politics of Media Policy. Cambridge: Polity Press.

García Canclini. (2001). Por qué legislar sobre industrias culturales. Recuperado de http://nuso.org/articulo/por-que-legislar-sobre-industrias-culturales/ 
Garnham, N. (2000). Emancipation, the media and Modernity. Arguments about the Media and Social Theory. Oxford: University Press.

Gómez, G. (2011). Gobiernos progresistas y políticas públicas de comunicación: una aproximación regional para provocar la reflexión. En A. Koschützke \& E. Gerber (Eds.), Progresismo y políticas de comunicación. Manos a la obra (pp. 15-32). Buenos Aires: Fundación Friedrich Ebert. Recuperado de http://library.fes.de/pdf-files/bueros/argentinien/08966.pdf

Jenkins, H. (2008). La cultura de la convergencia de los medios de comunicación. Barcelona: Paidós.

Marino, S (2013). Políticas de comunicación del sector audiovisual: las paradojas de modelos divergentes con resultados congruentes (tesis doctoral). Universidad de Buenos Aires. Argentina.

Mastrini, G. \& Becerra, M. (2016). Convergencia consolida concentración en América Latina: en varios países, cuatro o menos empresas dominan el 95\% del mercado. Recuperado de http://www.observacom.org/convergencia-consolidaconcentracion-en-america-latina-en-varios-paises-cuatro-o-menos-empresasdominan-95-del-mercado-de-acceso-a-internet/

Mastrini, G. \& Marino, S. (2008). Al final del período. Los límites del progresismo. Políticas de comunicación durante el gobierno de Néstor Kirchner. Oficios Terrestres, 14(21), 180-193.

Mastrini, G., De Charras, D. \& Fariña, C. (2013). Nuevas formas de regulación internacional y su impacto en el ámbito latinoamericano. En G. Mastrini, A. Bizberge y D. de Charras (Eds.). Las políticas de comunicación en el siglo XXI. Nuevos y viejos desafios. Buenos Aires: La Crujía.

Mattelart, A. (2006). Diversidad cultural y mundialización. Barcelona: Paidós.

Mattelart, A. \& Mattelart, M. (1997). Historia de las teorías de la comunicación. Barcelona: Paidós.

McChesney, R. (2004). The Problem of the Media. The Problem of the Media: U.S. Communication Politics in the 21st Century. Nueva York. Monthly Review Press.

Ministerio de Tecnologías de la Información y las Comunicaciones de Colombia (2016). Mesas: Política pública, TV y contenidos audiovisuales. Recuperado de http:// micrositios.mintic.gov.co/mesas_politica_publica_tv_contenidos_audiovisuales/

Monje, D. (2013). Políticas del audiovisual en el marco de la integración regional mercosureña. Período 1991-2007 (tesis doctoral). Universidad Nacional de la Plata. Argentina. Recuperado de http://hdl.handle.net/10915/26307 
Mosco, V. (1996). The Political Economy of Communication. Londres: Sage.

Mosco, V. (2011). La economía política de la comunicación: una tradición viva. En L. Albornoz (Comp.), Poder, medios, cultura. Una mirada crítica desde la economía política de la comunicación. Buenos Aires: Paidós.

Organización de las Naciones Unidas para la Educación, la Ciencia y la Cultura, UNESCO (2008). Indicadores de desarrollo mediático. Marco para evaluar el desarrollo de los medios de comunicación social. Recuperado de http://www. unesco.org/new/es/communication-and-information/resources/publications-andcommunication-materials/publications/full-list/media-development-indicators-aframework-for-assessing-media-development/

Pérez, D. (2014). “Documento Borrador. Propuesta para la actualización de los lineamientos de política de comunicación y cultura”. Recuperado de http://www.mincultura. gov.co/areas/comunicaciones/politicas-en-comunicacion-y-cultura/Documents/ Propuesta\%20para\%201a\%20actualizaci\%C3\%B3n\%20de\%201os\%20 lineamientos $\% 20 \mathrm{de} \% 20$ pol $\%$ C3\%ADtica $\% 20 \mathrm{de} \% 20$ comunicaci $\% \mathrm{C} 3 \% \mathrm{~B} 3 \mathrm{n} \% 20$ y\%20cultura.pdf

Pérez, D. (2015). "Lineamientos de la Dirección de Comunicaciones del Ministerio de Cultura". Recuperado de http://www.mincultura.gov.co/areas/comunicaciones/ politicas-en-comunicacion-y-cultura/Documents/Lineamientos $\% 20 \mathrm{de} \% 201 \mathrm{a} \% 20$ Direcci\%C3\%B3n\%20de\%20Comunicaciones.pdf

Rincón, O. (2010). La obsesión porque nos amen: crisis del periodismo/éxitos de los telepresidentes. En Amado, A. (Ed.), La palabra empeñada: investigaciones sobre medios y comunicación pública en Argentina (pp. 13-16). Buenos Aires: Friedrich Ebert Stiftung.

Saintout, F. \& Varela, A. (2013). Comunicación y gobiernos populares en América Latina. Cuadernos del Pensamiento Crítico Latinoamericano, 8, 2-3. Recuperado de http://biblioteca.clacso.edu.ar/clacso/se/20131227030015/CuadernoNo8.pdf

Santander, P. (2014). Nuevas leyes de medios en Sudamérica: enfrentando políticamente la concentración mediática. Convergencia, 21, 13-37.

Segura, S. (2011). Las disputas por democratizar las comunicaciones. Las tomas de posición de las organizaciones sociales (Córdoba, 2001-2009) (tesis doctoral). Universidad de Buenos Aires. Argentina.

Sierra, F. (2011). Consumo cultural y poder mediático. En L. Albornoz (Comp.), Poder, medios, cultura. Una mirada crítica desde la economía política de la comunicación. Buenos Aires: Paidós. 
Téllez, M. (2016). Políticas de comunicación y cultura en Colombia. Convergencias y divergencias. Bogotá: Pontificia Universidad Javeriana, Ministerio de Cultura. Recuperado de http://www.mincultura.gov.co/areas/comunicaciones/politicas-encomunicacion-y-cultura/politicacomunicacionycultura/Paginas/default.aspx

Téllez, M. (2003). La televisión comunitaria en Colombia. Entre la realidad y la utopía. Bogotá: Ministerio de Cultura, CNTV.

Waisbord, S. \& Segura, M. (2016). En la sociedad civil y reformas a las políticas de los medios de comunicación en América Latina. Recuperado de http://www. observacom.org/como-los-movimientos-sociales-han-cambiado-las-politicas-delos-medios-de-comunicacion-en-america-latina/

Wolhers, M. (2008). Convergencia tecnológica y agenda regulatoria de las telecomunicaciones en América Latina. Santiago de Chile: Naciones Unidas. Recuperado de https://core.ac.uk/download/pdf/38671919.pdf

Wolton, D. (2003). L'Autre mondialisation. París: Flammarion. 